\title{
Novel risk scoring system for immune checkpoint inhibitors treatment in non-small cell lung cancer
}

\author{
Chuling Li ${ }^{1 \#}$, Meiqi Shi" ${ }^{2 \#}$ Xinqing Lin ${ }^{3 \#}$, Yongchang Zhang ${ }^{4 \#}$, Shaorong Yu$^{2}$, Chengzhi Zhou ${ }^{3}$, \\ Nong Yang ${ }^{4}$, Jianya Zhang ${ }^{5}$, Fang Zhang ${ }^{5}$, Tangfeng Lv ${ }^{5}$, Hongbing Liu ${ }^{5}$, Yong Song ${ }^{1,5}$ \\ ${ }^{1}$ Department of Respiratory Medicine, Jinling Hospital, Nanjing Medical University, Nanjing, China; ${ }^{2}$ Department of Medical Oncology, The \\ Affiliated Cancer Hospital of Nanjing Medical University, Jiangsu Cancer Hospital, Jiangsu Institute of Cancer Research, Nanjing, China; ${ }^{3}$ State \\ Key Laboratory of Respiratory Disease, National Clinical Research Center for Respiratory Disease, Guangzhou Institute of Respiratory Health, \\ The First Affiliated Hospital of Guangzhou Medical University, Guangzhou, China; ${ }^{4}$ Department of Medical Oncology, Lung Cancer and \\ Gastrointestinal Unit, Hunan Cancer Hospital/The affiliated Cancer Hospital of Xiangya School of Medicine, Central South University, Changsha, \\ China; ${ }^{5}$ Department of Respiratory Medicine, Jinling Hospital, Nanjing University School of Medicine, Nanjing, China \\ Contributions: (I) Concept and design: C Li, M Shi, H Liu, Y Song; (II) Administrative support: C Li, Y Zhang, J Zhang, F Zhang; (III) Provision \\ of study materials or patients: C Li, Y Zhang, N Yang; (IV) Collection and assembly of data: M Shi, X Lin, C Zhou, H Liu; (V) Data analysis and \\ interpretation: C Li, M Shi, X Lin, S Yu, T Lv, H Liu; (VI) Manuscript writing: All authors; (VII) Final approval of manuscript: All authors. \\ "These authors contributed equally to this work. \\ Correspondence to: Yong Song, MD; Hongbing Liu, MD. East Zhongshan Road 305, 211100 Nanjing, China. Email: yongsong6310@yahoo.com; \\ netlhb@126.com.
}

Background: Immune checkpoint inhibitor (ICI)-based immunotherapy has improved the clinical outcome of non-small cell lung cancer (NSCLC). However, current indicators, such as programmed cell death-ligand 1 (PD-L1) expression in tumors or tumor mutational burden (TMB), are not considered ideal biomarkers for prognosis. Thus, there is an urgent requirement for a comprehensive risk scoring system.

Methods: In this study, we enrolled 464 NSCLC patients who received ICIs between March 2017 and January 2020 at four clinical centers. Univariate and multivariate (the logistic and the Cox regression) analyses were conducted to screen clinically relevant variables. Significant parameters $(\mathrm{P}<0.05)$ including absolute lymphocyte count (ALC, L), Eastern Cooperative Oncology Group Performance Status (ECOG PS, E) and lung/pleural metastasis (M) were selected for LEM score. Weighted values based on odds ratio and hazard ratio of multiple analyses were assigned to each parameter. LEM score was the sum of weighted values of each variable (Good, 0-1; Intermediate, 2-3; Poor, 4-6). Kaplan-Meier curves were used to evaluate the association between LEM score and progression-free survival (PFS).

Results: In total, 258 patients were pooled and stratified into three risk categories based on the LEM score. Objective response rate (ORR) was significantly higher in the good-risk group compared with the poorrisk group [55.9\% vs. $7.3 \%$, odds ratio $(\mathrm{OR}), 0.023$; 95\% confidence interval (CI), 0.005-0.099; $\mathrm{P}<0.001]$. Patients with good risk [hazard ratio (HR), 0.130; 95\% CI, 0.084-0.203; median PFS, 12.5 months; $\mathrm{P}<0.001$ ] or intermediate risk (HR, 0.330; 95\% CI, 0.222-0.490; median PFS, 4.2 months; $\mathrm{P}<0.001$ ) had longer PFS than those with poor risk (median PFS, 2.1 months). DNA sequencing was performed in 41 patients [no durable benefit (NDB): $n=29$; durable clinical benefit (DCB): $n=12$ ] and epidermal growth factor receptor (EGFR) mutations were enriched in samples of the NDB group vs. the DCB group (11/29 vs. 1/12; Fisher's exact $\mathrm{P}=0.073$; OR, 6.722; 95\% CI, 0.760-59.479). Additionally, patients with EGFR mutations had higher LEM scores than those with wild-type EGFR.

Conclusions: In conclusion, the LEM score provided a potential prognostic biomarker for NSCLC patients treated with ICIs.

Keywords: Non-small cell lung cancer (NSCLC); immune checkpoint inhibitors (ICIs); LEM; risk scoring system; prognostic biomarker 
Submitted Jul 08, 2020. Accepted for publication Dec 17, 2020.

doi: $10.21037 /$ tlcr-20-832

View this article at: http://dx.doi.org/10.21037/tlcr-20-832

\section{Introduction}

In the past decade, immune checkpoint inhibitors (ICIs) have dramatically altered the management of non-small-cell lung cancer (NSCLC) (1-3). Consequently, programmed cell death-1 (PD-1) inhibitors have proven more effective than conventional chemotherapy for the treatment of metastatic NSCLC (3-6). Additionally, the combination of PD-1 inhibitors and chemotherapy has also resulted in improved outcomes of NSCLC $(7,8)$.

Despite the advances in ICIs therapy, some patients do not respond to ICIs. Some of the diagnostic tests performed for ICIs include Programmed cell death ligand-1 (PD-L1) immunohistochemistry (IHC) and tumor mutational burden (TMB); however, these have proven to be imperfect biomarkers. Studies have shown that several patients without PD-L1 expression did respond to ICIs, while those with PD-L1 expression did not $(3,9)$. TMB is another potential indicator, which represents the number of somatic mutations detected by DNA sequencing (10). The lack of uniform methodology limits its widespread application. Therefore, there is an urgent need to develop a robust and reproducible scoring system to predict ICIs' response.

Several studies have tried to predict ICIs' response using various parameters, such as clinical features [e.g., metastatic site (11), computational image-based features $(12,13)]$, laboratory parameters [e.g., neutrophil-to-lymphocyte ratio (NLR) (14-16), lactate dehydrogenase (LDH) (17), tumor markers (18)], and genetic landscape $(19,20)$. Most of these parameters have yielded a poor performance due to lack of comprehensive evaluation in risk stratification. Recent studies have shown that the anti-tumor response to ICIs is a complicated process involving several factors. Previous studies have developed various prognostic models for prognostic evaluation in ICIs therapy. For example, a lung immune prognostic index (LIPI) combining derived NLR (dNLR) and LDH (21), as well as a risk scoring criteria including monocyte-to-lymphocyte ratio (MLR), sites of metastasis, and nutritional index-body mass index (BMI) (22) were respectively developed for NSCLC and metastatic renal cell cancer (mRCC) patients treated with ICIs.

Thus, we generated a novel risk scoring system for ICIs treatment in NSCLC. This index was labeled "LEM" and included absolute lymphocyte count (ALC) $<1.5 \times 10^{9} / \mathrm{L}$, Eastern Cooperative Oncology Group Performance Status (ECOG PS) $\geq 2$, and lung/pleura metastasis. Patients who received $\mathrm{PD}-1$ inhibitors were stratified into three risk stratifications (good, intermediate, and poor) based on the LEM score. Here, we developed this scoring system to explore the association between LEM score and clinical outcome.

We present the following article in accordance with the TRIPOD reporting checklist (available at http://dx.doi. org/10.21037/tlcr-20-832).

\section{Methods}

\section{Patient selection}

This study retrospectively screened 464 metastatic NSCLC patients who had been treated with PD-1 inhibitors [pembrolizumab (Merck Sharp \& Dohme), nivolumab (Bristol-Myers Squibb), sintilimab (Innovent), or toripalimab (Topalliance)] between March 2017 and January 2020 at four clinical centers. We excluded the patients who received initial PD-1 immunotherapy at out hospital but was lost-to-follow-up thereafter $(\mathrm{n}=110)$ and those who do not receive sufficient sessions for evaluation at the time of study $(\mathrm{n}=79)$. The final analysis included 258 patients. Data from Jingling Hospital ( $n=87$ ) were used to develop the risk scoring system, and the remaining data were used for validation (Figure S1).

Prior to treatment, we obtained clinical information and routine laboratory test records. Laboratory tests included blood platelet count, lymphocyte count, neutrophil count, NLR, levels of albumin, globulin, LDH and albumin to globulin ratio (A/G). Clinical data included demographic information, ECOG PS, TNM stage, metastatic site, and the number of prior therapies. PD-L1 (Dako 22C3) IHC staining was performed at the pathology department of each center. Response and progression were evaluated based on the RECIST v1.1 criterion (23). Patients were stratified as a durable clinical benefit (DCB: partial or stable response lasting $>6$ months) and no durable benefit (NDB) groups based on the published metrics $(19,24)$. The primary endpoint was progression-free survival (PFS) (time from 
initial ICIs administration to confirmed progressive disease radiologically or death due to any cause). We also measured other objectives including treatment efficacy (DCB/NDB), objective response rate (ORR) and one-year overall survival (OS) (time from initial ICIs administration to death due to any cause) rate.

Sintilimab and toripalimab are both domestic PD-1 inhibitors which have not yet been approved by FDA for NSCLC treatment. In the study, patients who received sintilimab or toripalimab were undergoing approved clinical trials. All procedures performed in this study were in accordance with the Declaration of Helsinki (as revised in 2013) and approved by the local ethics committee of Jinling Hospital (registration ID. 2017NZHX-022). Informed consent from individuals was waived based on the retrospective nature of this study.

\section{DNA sequencing and data sources}

Tumor tissue and corresponding blood samples $(n=41)$ collected from Jinling Hospital were sent to a gene company for DNA extraction and sequencing. DNA was extracted from the blood samples, and formalin-fixed paraffin embedded (FFPE) slices were prepared. Deep sequencing (139 genes, 10,000x) based on Illumina Hiseq2000 system platform (Illumina, USA) was used to detect gene alterations, such as missense mutation, insertion, deletion, copy number variation, etc. TMB was expressed as the number of non-synonymous mutations.

To further explore the genetic factors that influenced the clinical efficacy of ICIs, we compared the gene expression data with Helmann (19) (MSK, Cancer Cell 2018) (n=75) and Rizvi (25) (MSKCC, J Clin Oncol 2018) (n=240). The genomic information of NSCLC cohorts treated with ICIs was shared in the cBioPortal (www. cbioportal.org).

\section{Statistical analysis}

The Mann-Whitney $U$ test was used for univariate analyses. The logistic regression model and the Cox regression model were used to evaluate the association between characteristics and best response/PFS. After multivariate analyses, factors of statistical significance were included in the risk scoring system, and the weight values of all indices were determined based on the odds ratio (OR) for response and hazard ratio (HR) for PFS. Survival estimates were generated by KaplanMeier analysis, and the log-rank test was used to compare the differences in PFS among subgroups. Statistical analysis was done using the SPSS software (v22.0, SPSS, Inc., Chicago) and expressed using GraphPad Prism 5 and R 3.3.2 software. A two-sided $\mathrm{P}<0.05$ was considered statistically significant.

\section{Results}

\section{Baseline clinical features of the cohort}

Table 1 summarizes the baseline characteristics of 258 NSCLC patients. In the pooled cohort, the median age of patients was 61 years (range, 38-81); 200 (77.5\%) patients were male; $150(58.1 \%)$ patients had a history of smoking. Of the 258 NSCLC patients, 88 (34.1\%) had the squamous subtype, and $170(65.9 \%)$ had the non-squamous subtype. Most patients (81.4\%) were in good physical health with ECOG PS $<2$. Approximately $121(47.6 \%)$ patients had contralateral lung or pleura metastasis, while 27 (10.6\%) had liver metastasis. Additionally, 150 (58.1\%) patients received PD-1 inhibitors as the first-line or second-line of treatment. Of the 258 patients enrolled in this study, 54 were treated with PD-1 inhibitor monotherapy, while others received a combination treatment of PD-1 inhibitor and chemotherapy.

\section{Univariate and multivariate analyses of baseline characteristics}

The univariate analyses of routine laboratory parameters revealed that there was a significant difference in the levels of ALC $\left[(1.191 \pm 0.068) \times 10^{9} / \mathrm{L} v s .(1.787 \pm 0.122) \times 10^{9} / \mathrm{L}\right.$; $\mathrm{P}<0.001$ ] (Figure $\mathrm{S} 2 \mathrm{~A}), \mathrm{NLR}(5.410 \pm 0.679$ vs. $3.717 \pm 0.373$; $\mathrm{P}<0.05)$ (Figure $\mathrm{S} 2 \mathrm{~B})$, albumin level $(35.48 \pm 0.637$ vs. $37.40 \pm 0.768 \mathrm{~g} / \mathrm{L} ; \mathrm{P}<0.05$ ) (Figure $\mathrm{S} 2 \mathrm{C}$ ), and $\mathrm{A} / \mathrm{G}$ ratio $(1.185 \pm 0.047$ vs. $1.309 \pm 0.036 ; \mathrm{P}<0.05)$ (Figure $\mathrm{S} 2 \mathrm{D}$ ) in the DCB and NDB groups. However, we found no significant difference for other parameters among the groups (Figure S3).

After univariate analyses, marginally significant $(\mathrm{P}<0.1)$ factors and demographic characteristics were included for multivariate analyses (Table 2). The ORR was $36.8 \%$ implying that of the 87 patients, 32 responded. Response to ICIs treatment was associated with ECOG PS [OR, 5.242; 95\% confidence interval (CI), 1.056-26.016; $\mathrm{P}=0.043$ ], ALC (OR, 0.197; 95\% CI, 0.071-0.545; P=0.002), and lung/pleura metastasis (OR, 3.638; 95\% CI, 1.090-12.143; $\mathrm{P}=0.036)$. Cox regression model revealed that inferior PFS was associated with ECOG PS $\geq 2$ (HR, 2.312; 95\% CI, 
Table 1 Baseline characteristics of patients

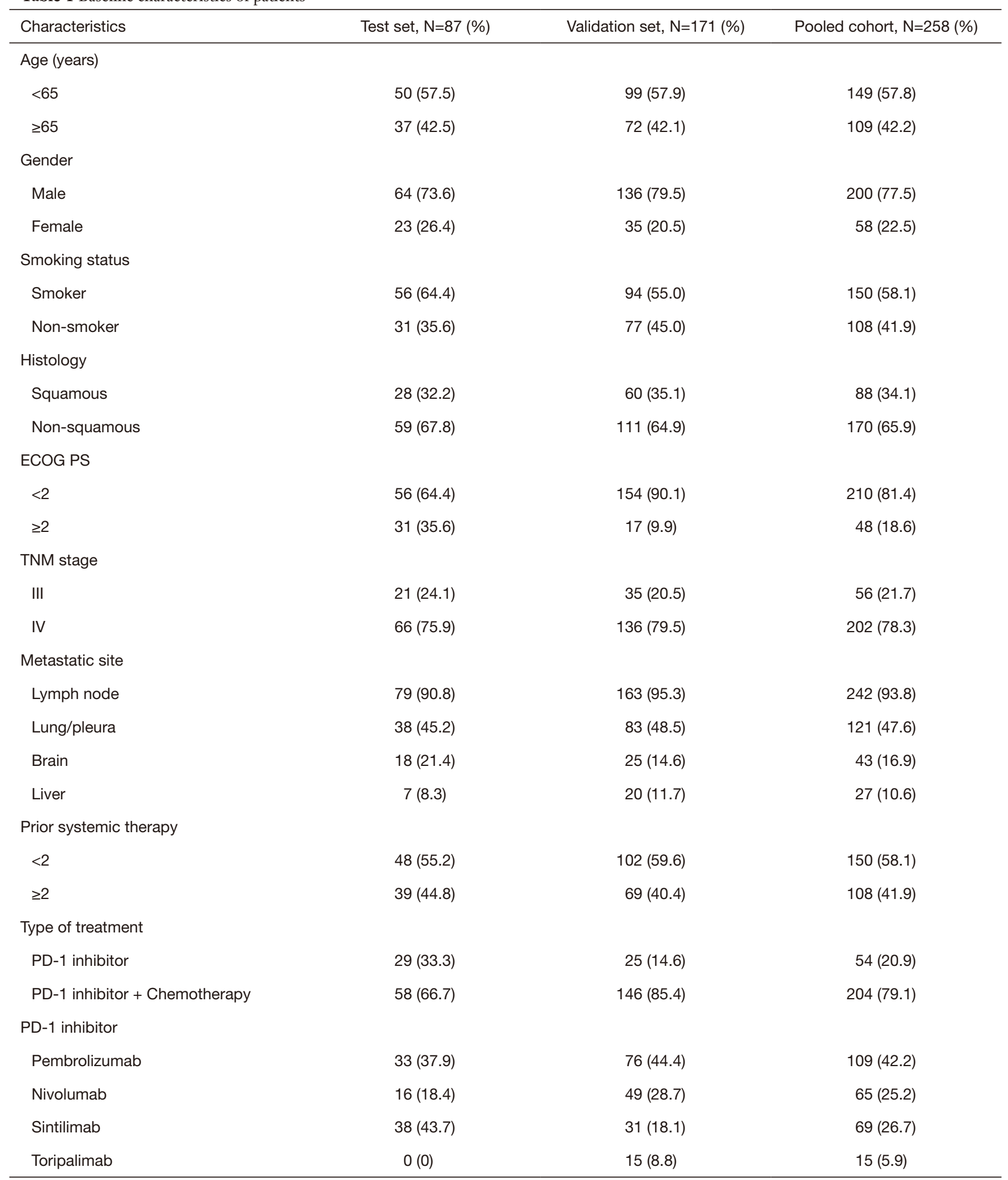

Table 1 (continued) 
Table 1 (continued)

\begin{tabular}{|c|c|c|c|}
\hline Characteristics & Test set, $\mathrm{N}=87$ (\%) & Validation set, $\mathrm{N}=171$ (\%) & Pooled cohort, $\mathrm{N}=258(\%)$ \\
\hline EGFR mutation & $14(18.4)$ & $18(15.9)$ & $32(16.9)$ \\
\hline ALK rearrangement & $2(2.6)$ & $5(4.4)$ & $7(3.7)$ \\
\hline Unknown & $10(11.5)$ & $58(33.9)$ & $69(26.7)$ \\
\hline Negative & $13(14.9)$ & $21(12.3)$ & $34(13.2)$ \\
\hline Positive & $26(29.9)$ & $34(19.9)$ & $60(23.2)$ \\
\hline Unknown & $48(55.2)$ & $116(67.8)$ & $164(63.6)$ \\
\hline \multicolumn{4}{|l|}{ Best response } \\
\hline PFS, median, month (95\% Cl) & $5.125(3.834-6.416)$ & $6.637(3.643-9.631)$ & $5.585(3.971-7.199)$ \\
\hline
\end{tabular}

ALK, anaplastic lymphoma kinase; $\mathrm{Cl}$, confidence interval; CR, complete response; ECOG PS, Eastern Cooperative Oncology Group Performance Status; EGFR, epidermal growth factor receptor; HR, hazard ratio; PD, progression disease; PFS, progression-free survival; $\mathrm{PR}$, partial response; SD, stable disease.

Table 2 Multiple logistic regression analysis for response and Cox regression analysis for progression-free survival (PFS) in the test set

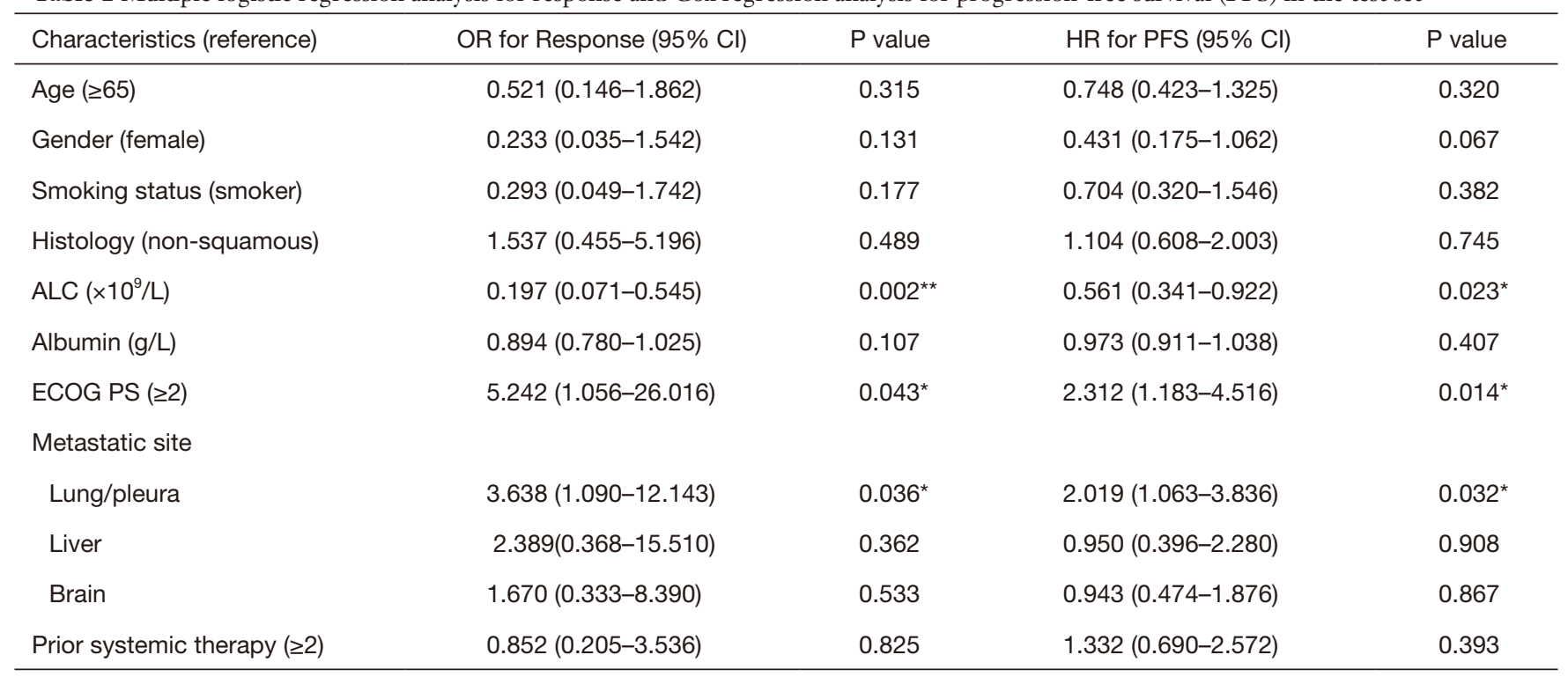

ALC, absolute lymphocyte count; ECOG PS, Eastern Cooperative Oncology Group Performance Status; HR, hazard ratio; OR, odds ratio; PFS, progression-free survival; ${ }^{*}, \mathrm{P}<0.05 ;{ }^{* \star}, \mathrm{P}<0.01$.

1.183-4.516; $\mathrm{P}=0.014)$ and lung/pleura metastasis (HR, 2.019; 95\% CI, 1.063-3.836; $\mathrm{P}=0.032)$. However, patients with higher ALC had significantly longer PFS (HR, 0.561; 95\% CI, 0.341-0.922; $\mathrm{P}=0.023$ ).

\section{Analyses of the LEM risk scoring system}

The final selected variables were ALC, ECOG PS and lung/ pleura metastasis. Relative weights were based on odds ratio and hazard ratio of multivariate analyses (high HR/OR: 
Table 3 The LEM prognostic scoring system and risk stratification construction

\begin{tabular}{|c|c|c|c|c|c|c|}
\hline Variable & \multicolumn{2}{|c|}{ Absolute lymphocyte count $\left(\times 10^{9} / L\right)$} & \multicolumn{2}{|c|}{ Lung/pleura metastasis } & \multicolumn{2}{|c|}{ ECOG PS } \\
\hline Weighted value & 1 & 0 & 2 & 0 & 3 & 0 \\
\hline Risk stratification (LEM score) & \multicolumn{6}{|c|}{ Good [0-1]; intermediate [2-3]; poor [4-6] } \\
\hline
\end{tabular}

Weighted values based on odds ratio and hazard ratio of multiple analyses (high HR/OR: weighted value =3; intermediate HR/OR: weighted value $=2$; low HR/OR: weighted value $=1$ ) were assigned to each parameter. LEM score was the sum of weighted values of each variable. ECOG PS, Eastern Cooperative Oncology Group Performance Status.
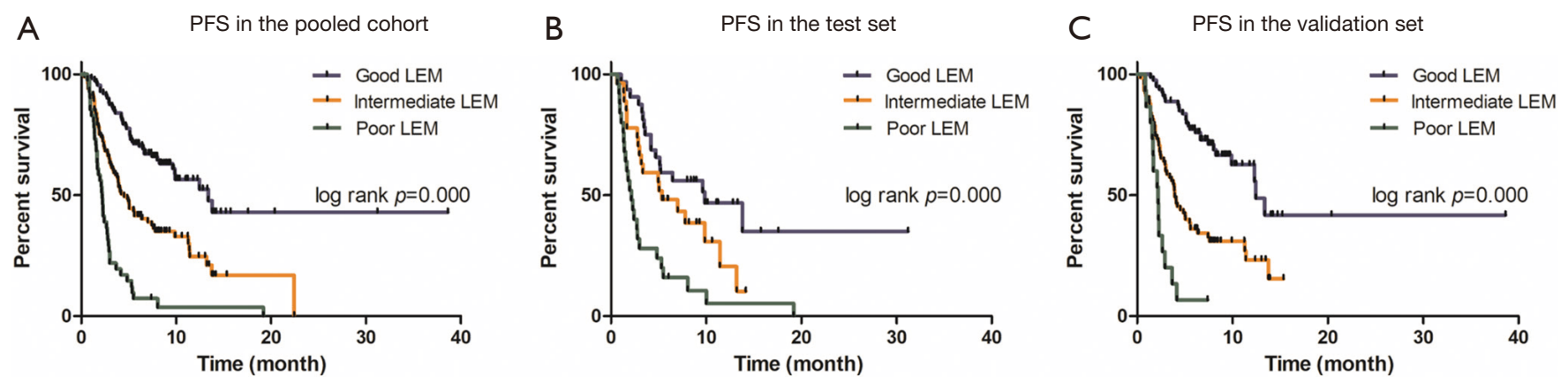

Figure 1 Progression-free survival (PFS) based on the LEM score. (A) The pooled cohort $(\mathrm{P}<0.001)$; (B) The test set $(\mathrm{P}<0.001)$; $(\mathrm{C})$ The validation set $(\mathrm{P}<0.001)$. LEM score: Good: 0-1; Intermediate: 2-3; Poor: 4-6.

weighted value $=3$; intermediate $\mathrm{HR} / \mathrm{OR}$ : weighted value =2; low HR/OR: weighted value $=1$ ). Weighted values were assigned to each parameter, and LEM score was the sum of weighted values of each variable (Table 3).

In the test set, based on the LEM score, patients were divided into three risk groups [good $(37.9 \%)$, intermediate (32.2\%), and poor (29.9\%)]. We found that a good risk (HR, 0.216; 95\% CI, 0.117-0.398; median PFS, 9.9 months; $\mathrm{P}<0.001)$ and an intermediate risk (HR, 0.322; 95\% CI, 0.176-0.591; median PFS, 7.0 months; $\mathrm{P}<0.001$ ) was associated with longer PFS compared with a poor risk (median PFS, 2.1 months). The validation set further verified these results $[\operatorname{good}(45.6 \%)$, intermediate $(45.6 \%)$, and poor $(8.8 \%)$ ]. Patients with good risk (HR, 0.076; 95\% CI, 0.038-0.150; median PFS, 12.5 months; $\mathrm{P}<0.001$ ) or intermediate risk (HR, 0.240; 95\% CI, 0.130-0.443; median PFS, 3.9 months; $\mathrm{P}<0.001)$ trended toward longer PFS than those with poor risk (median PFS, 2.1 months) (Figure 1 and Table 4). Further subgroup analyses based on smoking status, age, and histology revealed similar association between LEM score and PFS (log-rank $\mathrm{P}<0.001$ ) (Figure 2). Figure $\mathrm{S} 4$ also shows the one-year OS rate based on the LEM score.
We found that LEM score was associated with ORR $(\mathrm{P}<0.001)$, and ORR ranged from $7.7 \%$ (for poor risk) to $54.5 \%$ (for good risk) in the test set. The pooled cohort also showed significant difference in ORR: 7.3\% (for poor risk) vs. 55.9\% (for good risk) (OR, 0.023; 95\% CI, 0.005-0.099; $\mathrm{P}<0.001)$ and $33.0 \%$ (for intermediate risk) (OR, 0.078; 95\% CI, 0.005-0.099; $\mathrm{P}<0.001$ ) (Table S1).

\section{Association between gene mutation and LEM score}

Tumor tissue from 41 patients (NDB: $n=29$; DCB: $n=12$ ) were processed for DNA sequencing. We limited our research to 34 genes which were either highly deleterious or had mutations in at least three patients. These variants were used for further analysis. Figure 3 shows the gene profiles (frameshift insertion or deletion, splice-site, or missense mutation) for the two groups, and Table S2 shows the corresponding LEM scores.

The most frequent mutations detected were TP53 $(\mathrm{n}=27,65.9 \%)$, EGFR $(\mathrm{n}=12,29.3 \%)$, KRAS $(\mathrm{n}=11$, $26.8 \%)$, and PIK3CA ( $n=8,19.5 \%)$. The distribution of genetic alterations in KRAS (NDB: 6/29 vs. DCB: 5/12), PIK3CA (NDB: 6/29 vs. DCB: $2 / 12$ ) and TP53 (NDB: 


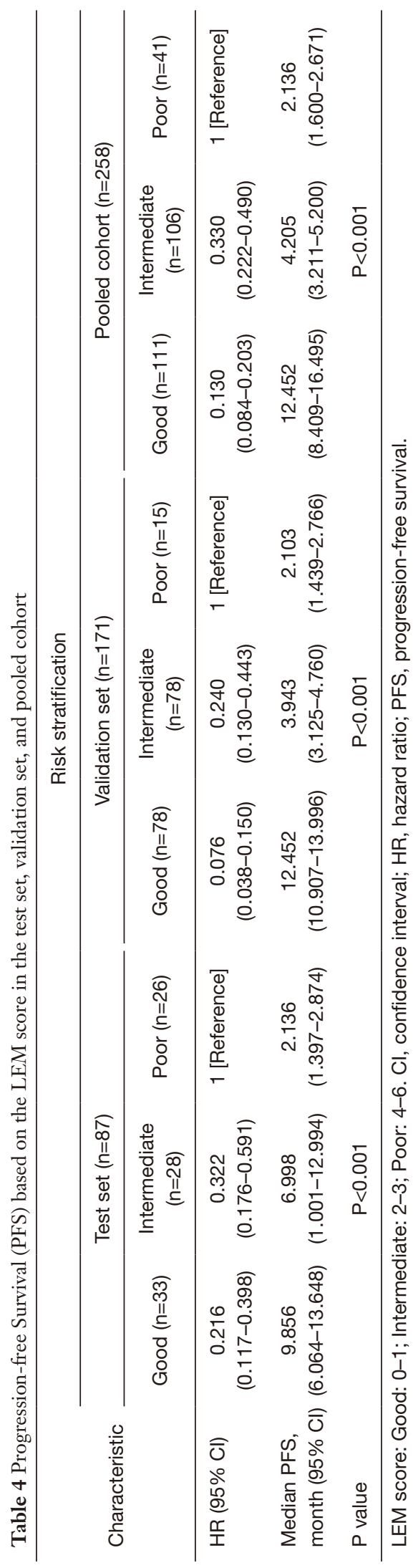

20/29 vs. DCB: 7/12) was similar for the NDB/DCB group. Nevertheless, mutations in EGFR (ex19del: 6/12; ex20ins: 5/12; ex21L858R: 1/12) were enriched in samples of the NDB group vs. the DCB group with negligible significance (11/29 vs. $1 / 12$; Fisher's exact $\mathrm{P}=0.073$; OR, $6.722 ; 95 \%$ CI, 0.760-59.479). Here, mutations in ARID2, CCNE1, CDKN2A, MET, PKHD1, SETD and RAF1 were all observed in the NDB group. Additionally, mutations in LRP1B, NTRK3 and TERT in the DCB group were more than that in the NDB group.

Next, we cross-validated our data with the published datasets to verify our findings. Datasets from Rizvi (25) $(\mathrm{n}=240)$ and Helmann (19) $(\mathrm{n}=75)$ contained both genomic data and clinical response (NDB $v s$. DCB) to ICIs treatment in NSCLC. It showed that EGFR mutations were related to ICIs treatment response in both our cohort $(\mathrm{P}=0.004)$ as well as datasets of Rizvi and Helmann $(\mathrm{P}=0.036)$. Additionally, in Rizvi and Helmann's dataset, mutations in AR $(\mathrm{P}=0.036)$, FAT1 $(\mathrm{P}=0.036)$ and KMT2C $(\mathrm{P}=0.036)$ trended toward DCB, while in our cohort, TP53 mutations were associated with NDB $(\mathrm{P}=0.029)$ (Figure $4 A)$. Due to the small sample size of our study, we further combined our data with these two datasets. Mutations in FAT1 (OR, 0.502; $\mathrm{P}=0.087)$, FBXW7 (OR, 0.342; $\mathrm{P}=0.087), \mathrm{KMT} 2 \mathrm{C}(\mathrm{OR}, 0.5$; $\mathrm{P}=0.075)$, and STK11 $(\mathrm{OR}, 1.766 ; \mathrm{P}=0.082)$ were associated with treatment response with negligible significance; however, EGFR mutation (OR, 3.149; $\mathrm{P}=0.001)$ showed statistically significance (Figure 4B).

We further explored the association between individual EGFR mutations and LEM scores. Data derived from our pooled set showed that LEM scores of patients with EGFR mutations had a higher LEM score than those with wildtype EGFR $\left[(3.000 \pm 0.359) \times 10^{9} / \mathrm{L} v s .(2.182 \pm 0.142) \times 10^{9} / \mathrm{L}\right.$; $\mathrm{P}=0.025$ ] (Figure $4 C$ ), while there was no difference among EGFR mutation sites (Ex19del, Ex20ins and Ex21L858R) (Figure 4D).

\section{Discussion}

ICIs therapy is considered a milestone in the history of NSCLC treatment. However, only some patients are benefitted due to the lack of comprehensive biomarkers $(10,26)$. Hence, there is an urgent need to develop a risk scoring system to stratify NSCLC patients. Thus, our study retrospectively investigated factors associated with ICIs treatment response to establish and verify a novel risk scoring system in four centers. Based on the results of univariate and multivariate analyses, the LEM score 

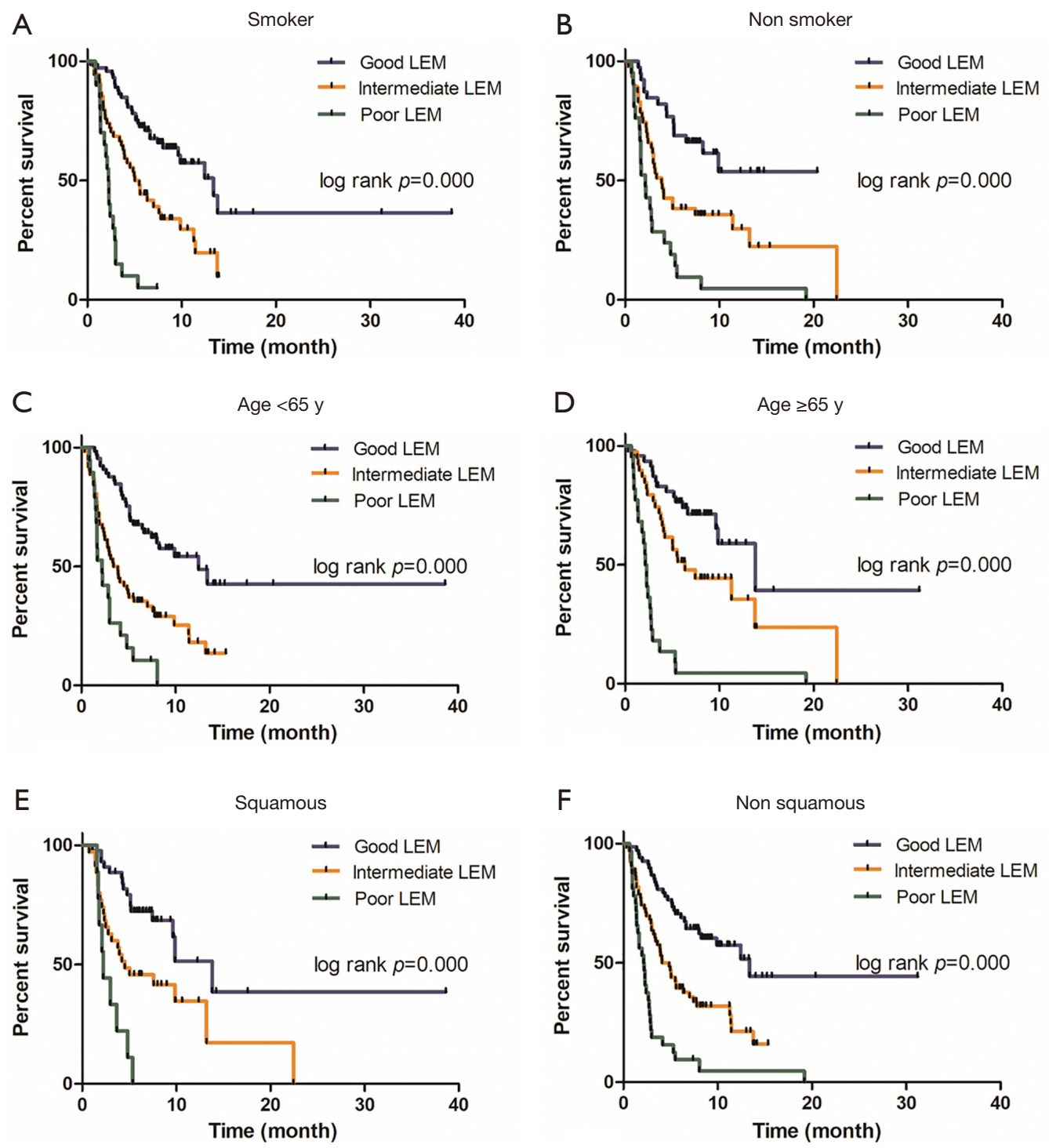

Figure 2 Subgroup analyses of progression-free survival (PFS) based on the LEM score in the pooled cohort based on the (A,B) smoking status (smoker vs. non-smoker, $\mathrm{P}<0.001)$; (C,D) age $(<65$ vs. $\geq 65, \mathrm{P}<0.001)$ and $(\mathrm{E}, \mathrm{F})$ histology (squamous vs. non-squamous, $\mathrm{P}<0.001)$. LEM score: Good: 0-1; Intermediate: 2-3; Poor: 4-6.

included ALC (L), ECOG PS (E), and lung/pleural metastasis status $(M)$. A higher LEM score was associated with limited response and inferior PFS, as well as EGFR mutation. Therefore, the LEM score could act as a pretreatment guide for optimization and candidate selection for ICIs therapy in NSCLC.

Metastatic sites are known to influence the efficacy of cancer treatment by formatting specific tumor microenvironment (TME) (27). Previous studies have shown the association between liver metastasis and a lower
ICIs treatment response rate $(11,28)$. Other studies have also shown that NSCLC patients with pleural metastasis experience more serious adverse events (SAEs), exhibit a limited response to ICIs (29), and have poor prognosis $(30,31)$. In this study, we observed that lung/pleura metastases but not liver or brain metastasis influenced ICIs treatment outcomes. Lung/pleura was the most common metastatic site of advanced NSCLC (32). Additionally, patients with EGFR mutations and anaplastic lymphoma kinase (ALK) rearrangements were more likely to have 


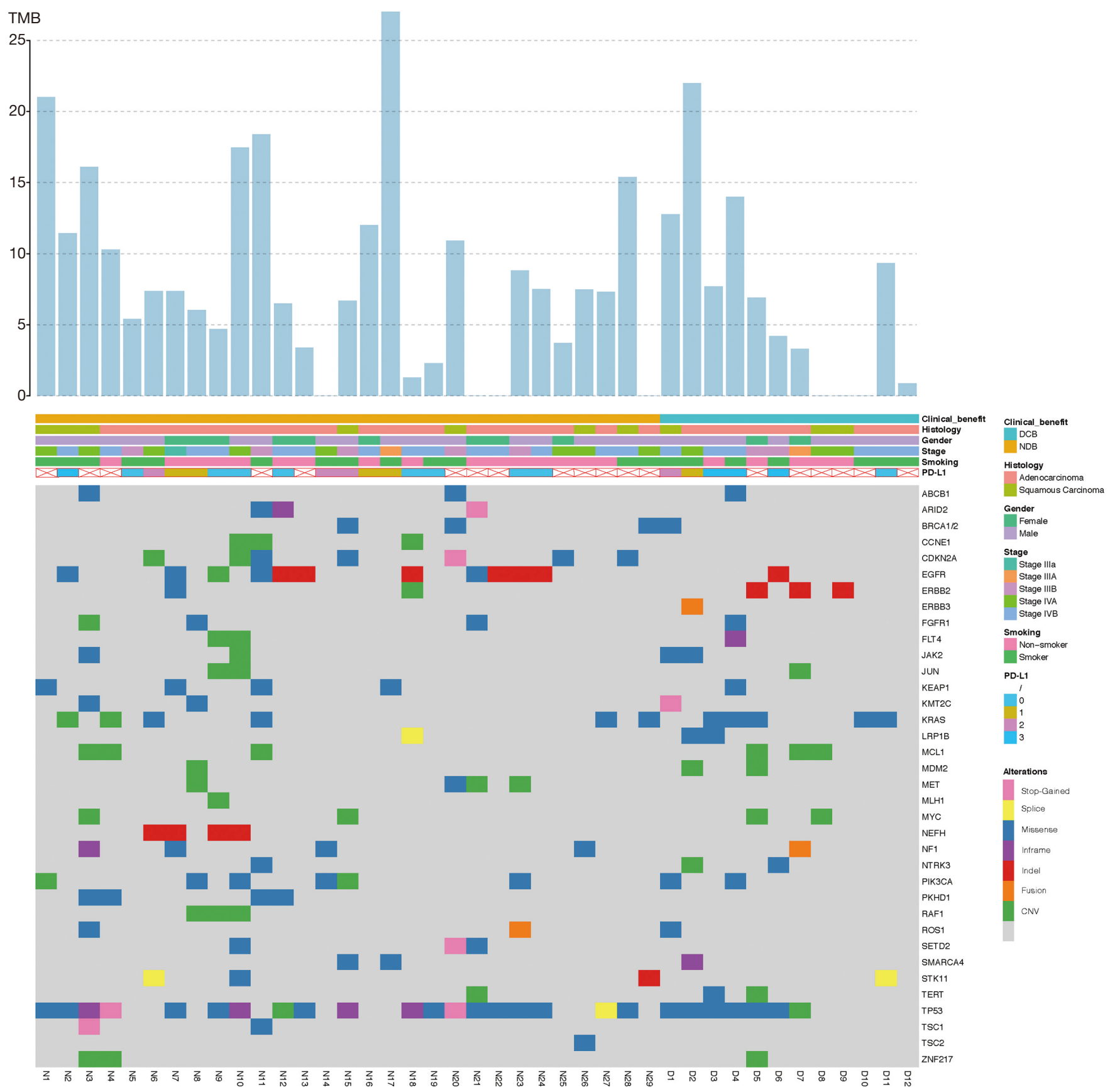

Figure 3 Gene alterations landscape of patients in the NDB and DCB groups. Thirty-four genes are shown, which were highly deleterious or had variants in at least three patients. Each column represents one patient. TMB value and clinical features are shown at the top. NDB (N): no durable benefit; DCB (D): durable clinical benefit.

metastasis to pleura and lung, respectively (33-35). The results of clinical trials, as well as our study, confirmed that EGFR mutations had a negative impact on ICIs treatment response (36). This might partially explain why lung/pleura metastasis was a negative factor in the current risk scoring system.

Systemic inflammatory and immune status are known to impact the efficacy of cancer treatments. Several blood parameters are used as ICIs biomarkers. Here, we found a correlation between ALC and ICIs treatment response. 
A

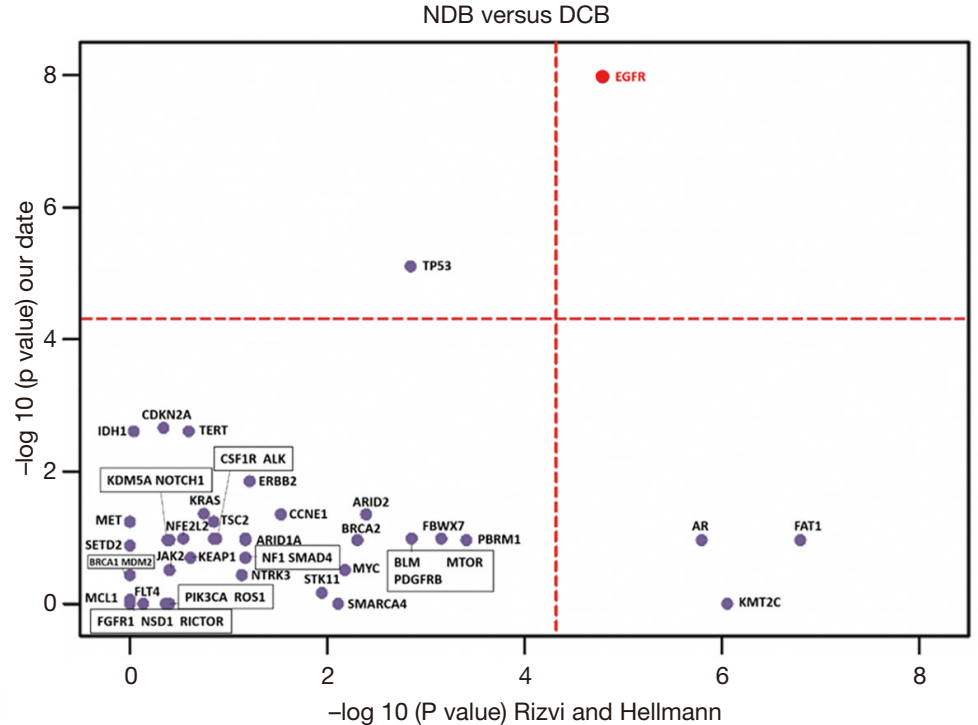

B

\begin{tabular}{|c|c|c|c|c|}
\hline EGFR & FAT1 & FBXW7 & KMT2C & STK11 \\
\hline 3.149 & 0.502 & 0.342 & 0.5 & 1.766 \\
\hline
\end{tabular}

C

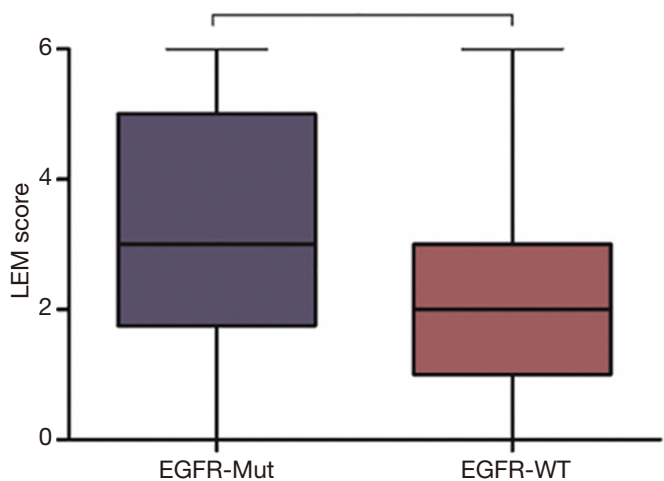

D

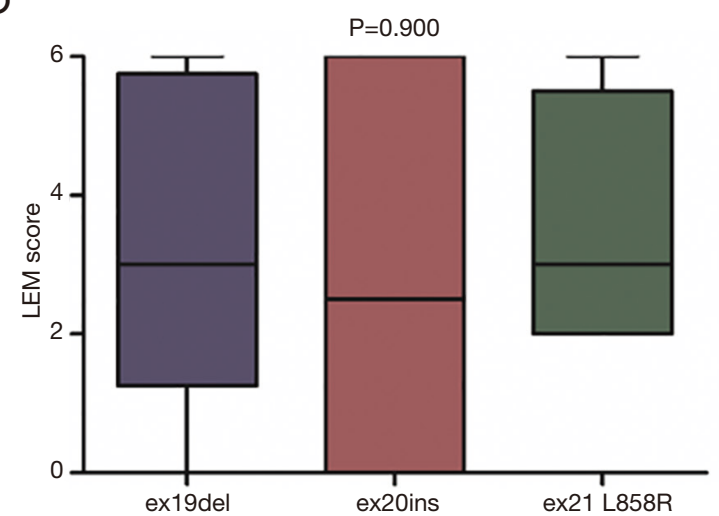

Figure 4 Gene alteration distribution associated with ICIs treatment response. (A) The comparison of gene alterations that were differentially expressed in the NDB/DCB group between our data and Rizvi and Hellmann datasets based on -log10 (P value). Red lines indicate $\mathrm{P}<0.05$. (B) $\mathrm{OR}$ values of gene alterations that were differentially expressed in the NDB/DCB group with negligible significance (FAT1, FBXW7, KMT2C, STK11 P<0.1; EGFR P<0.05) in combined datasets. Red and blue colors color indicate negative and positive factors for ICIs; (C) LEM scores differed between EGFR-mutated/wildtype groups, *, P<0.05. (D) LEM scores showed no significant difference among the EGFR mutation sites. Mut, mutated; WT, wildtype; ex19del: exon 19 deletions; ex20ins: exon 20 insertions; ex21L858R: exon 21 L858R.

PD-1 inhibitors are known to enhance the anti-tumor immunity of $\mathrm{T}$ lymphocytes by blocking PD-1 protein, which is expressed on the cell surface. The ICIs treatment focuses on advanced melanoma due to the presence of abundant lymphocytes (37). Thus, reduced levels of circulating lymphocytes might lead to a decrease in tumorinfiltrating T lymphocytes (TIL) as well as an imbalance in Th- 1 and Th-2 phenotypes $(38,39)$. Additionally, potential inflammatory biomarkers, including NLR, albumin, LDH, C-reactive protein (CRP), neutrophils, and platelets, have 
been shown to be associated with ICIs treatment response $(40,41)$. However, in our test cohort, we observed that NLR, albumin and $\mathrm{A} / \mathrm{G}$ ratio rather than $\mathrm{LDH}$, neutrophils, and platelets were associated with treatment outcomes. This difference might be attributed to the potential bias caused by the confounding factors in real-world data analysis.

Previous studies have shown that PD-L1 and TMB re correlated to clinical benefits of ICIs $(2,8,42)$. Hu-Lieskovan et al. (43) explored the association between PD-L1/TMB and benefit from pembrolizumab and discovered that PD-L1 was related to ORR [OR, $0.96(0.93-0.99), \mathrm{P}=0.007$ ] and PFS [HR, 0.98 (0.96-0.99), $\mathrm{P}=0.002$ ], while no such association was found for TMB. However, our novel scoring system showed great predictive value for both ORR [good risk: OR, $0.023(0.005-0.099) ; \mathrm{P}<0.001]$ and $\mathrm{PFS}$ [good risk: HR, $0.130(0.084-0.203) ; \mathrm{P}<0.001]$ even in the absence of PD-L1 and TMB.

Single parameters are known to have limited performance as a prognostic predictor. Previous studies have explored risk stratification score for patients treated with ICIs. Mezquita et al. (21) developed a prognostic index, LIPI, based on a multicenter retrospective study with a total of 466 ICIstreated NSCLC. This index based on dNLR greater than 3 and LDH greater than upper limit of normal (ULN) was correlated with worse outcomes for ICIs (good, 0 factor; intermediate, 1 factor; poor, 2 factors). Furthermore, it was further verified by Kazandjian (44) and Sorich (45). Mazzaschi et al. (46) also generated an Immune effector score $\left(\mathrm{I}_{\text {eff }} \mathrm{S}\right)$ featuring high soluble PD-L1 (sPD-L1) and low $\mathrm{CD} 8^{+} \mathrm{PD}-1^{+}$and NK cells levels, which outperformed LIPI in the prognostic power. It even showed remarkable impact of $\mathrm{I}_{\text {eff }} \mathrm{S}$ and LIPI integration on survival outcome. Kasahara et al. (47) employed Glasgow prognostic score (GPS), which contained CRP and albumin, to predict the efficacy of treatment with PD-1 inhibitors. These retrospective studies had certain limitations regarding the lack of comprehensive clinical and pathological data. Martini et al. (22) also developed a risk scoring criteria for patients with mRCC who were treated with ICIs. These criteria included MLR, sites of metastasis and nutritional index-BMI. Patients were also categorized into 4 groups (good, intermediate, poor, very poor). It turned out to be an effective way to predict survival in mRCC patients receiving ICIs. Also, another study (48) indicated that the ECOG PS, which reflected overall performance status, was better than BMI for risk stratification of survival in patients with metastatic cancer.

Our study had several limitations. First, it was a retrospective study based on real-world data; thus, there was scope for potential bias due to loss to follow-up or missing data. For example, in our study, PD-L1 IHC status and TMB were not routinely tested in our study, especially for those who underwent posterior-line ICIs treatment. Second, we chose a one-year OS rate rather than OS as the observe objective due to insufficient follow-up data. In future studies, we would investigate the association between LEM score and OS. Finally, only a few patients (41/258) had tumor sequencing data. Further efforts are needed to develop a more comprehensive index combining genomic and clinical variables to predict response to ICIs treatment.

\section{Conclusions}

Thus, the LEM score is a novel risk scoring system consisting of ALC (L), ECOG PS (E), and lung/pleural metastasis (M). It could act as a potential prognostic biomarker of ORR and PFS for patients treated with ICIs in NSCLC. Further large-sample studies are required to externally validate the LEM score.

\section{Acknowledgments}

The authors would like to thank all the reviewers who participated in the review and MJEditor (www.mjeditor. com) for its linguistic assistance during the preparation of this manuscript.

Funding: This work was supported by the National Natural Science Foundation of China (No. 81802301 and 81772500) and the Social Development Foundation of China (No. BE2019719).

\section{Footnote}

Reporting Checklist: The authors have completed the TRIPOD reporting checklist. Available at http://dx.doi. org/10.21037/tlcr-20-832

Data Sharing Statement: Available at http://dx.doi. org/10.21037/tlcr-20-832

Peer Review File: Available at http://dx.doi.org/10.21037/ tlcr-20-832

Conflicts of Interest: All authors have completed the ICMJE uniform disclosure form (available at http://dx.doi. org/10.21037/tlcr-20-832). YS and TL serve as unpaid editorial board members of Translational Lung Cancer 
Research. The other authors have no conflicts of interest to declare.

Ethical Statement: The authors are accountable for all aspects of the work in ensuring that questions related to the accuracy or integrity of any part of the work are appropriately investigated and resolved. All procedures performed in this study were in accordance with the Declaration of Helsinki (as revised in 2013) and approved by the local ethics committee of Jinling Hospital (registration ID. 2017NZHX-022). Informed consent from individuals was waived based on the retrospective nature of this study.

Open Access Statement: This is an Open Access article distributed in accordance with the Creative Commons Attribution-NonCommercial-NoDerivs 4.0 International License (CC BY-NC-ND 4.0), which permits the noncommercial replication and distribution of the article with the strict proviso that no changes or edits are made and the original work is properly cited (including links to both the formal publication through the relevant DOI and the license). See: https://creativecommons.org/licenses/by-nc-nd/4.0/.

\section{References}

1. Rittmeyer A, Barlesi F, Waterkamp D, et al. Atezolizumab versus docetaxel in patients with previously treated non-small-cell lung cancer (OAK): a phase 3, openlabel, multicentre randomised controlled trial. Lancet 2017;389:255-65.

2. Reck M, Rodriguez-Abreu D, Robinson AG, et al. Pembrolizumab versus Chemotherapy for PD-L1Positive Non-Small-Cell Lung Cancer. N Engl J Med 2016;375:1823-33.

3. Borghaei H, Paz-Ares L, Horn L, et al. Nivolumab versus Docetaxel in Advanced Nonsquamous Non-Small-Cell Lung Cancer. N Engl J Med 2015;373:1627-39.

4. Herbst RS, Baas P, Kim D-W, et al. Pembrolizumab versus docetaxel for previously treated, PD-L1-positive, advanced non-small-cell lung cancer (KEYNOTE-010): a randomised controlled trial. Lancet 2016;387:1540-50.

5. Brahmer J, Reckamp KL, Baas P, et al. Nivolumab versus Docetaxel in Advanced Squamous-Cell Non-Small-Cell Lung Cancer. N Engl J Med 2015;373:123-35.

6. Garon EB, Rizvi NA, Hui R, et al. Pembrolizumab for the treatment of non-small-cell lung cancer. N Engl J Med 2015;372:2018-28.

7. Langer CJ, Gadgeel SM, Borghaei H, et al. Carboplatin and pemetrexed with or without pembrolizumab for advanced, non-squamous non-small-cell lung cancer: a randomised, phase 2 cohort of the open-label KEYNOTE-021 study. Lancet Oncol 2016;17:1497-508.

8. Gandhi L, Rodriguez-Abreu D, Gadgeel S, et al. Pembrolizumab plus Chemotherapy in Metastatic NonSmall-Cell Lung Cancer. N Engl J Med 2018;378:2078-92.

9. Brahmer J, Reckamp KL, Baas P, et al. Nivolumab versus Docetaxel in Advanced Squamous-Cell Non-Small-Cell Lung Cancer. N Engl J Med 2015;373:123-35.

10. Doroshow DB, Sanmamed MF, Hastings K, et al. Immunotherapy in Non-Small Cell Lung Cancer: Facts and Hopes. Clin Cancer Res 2019;25:4592-602.

11. Tumeh PC, Hellmann MD, Hamid O, et al. Liver Metastasis and Treatment Outcome with Anti-PD-1 Monoclonal Antibody in Patients with Melanoma and NSCLC. Cancer Immunol Res 2017;5:417-24.

12. Tunali I, Gray JE, Qi J, et al. Novel clinical and radiomic predictors of rapid disease progression phenotypes among lung cancer patients treated with immunotherapy: An early report. Lung Cancer 2019;129:75-9.

13. van de Donk PP, Kist de Ruijter L, Lub-de Hooge MN, et al. Molecular imaging biomarkers for immune checkpoint inhibitor therapy. Theranostics 2020;10:1708-18.

14. Szor DJ, Dias AR, Pereira MA, et al. Prognostic Role of Neutrophil/Lymphocyte Ratio in Resected Gastric Cancer: A Systematic Review and Meta-analysis. Clinics (Sao Paulo) 2018;73:e360.

15. Paramanathan A, Saxena A, Morris DL. A systematic review and meta-analysis on the impact of pre-operative neutrophil lymphocyte ratio on long term outcomes after curative intent resection of solid tumours. Surg Oncol 2014;23:31-9.

16. Zer A, Sung MR, Walia P, et al. Correlation of Neutrophil to Lymphocyte Ratio and Absolute Neutrophil Count With Outcomes With PD-1 Axis Inhibitors in Patients With Advanced Non-Small-Cell Lung Cancer. Clin Lung Cancer 2018;19:426-34.e1.

17. Schiwitza A, Schildhaus HU, Zwerger B, et al. Monitoring efficacy of checkpoint inhibitor therapy in patients with non-small-cell lung cancer. Immunotherapy 2019;11:769-82.

18. Lang D, Horner A, Brehm E, et al. Early serum tumor marker dynamics predict progression-free and overall survival in single PD-1/PD-L1 inhibitor treated advanced NSCLC-A retrospective cohort study. Lung Cancer 2019;134:59-65.

19. Hellmann MD, Nathanson T, Rizvi H, et al. Genomic 
Features of Response to Combination Immunotherapy in Patients with Advanced Non-Small-Cell Lung Cancer. Cancer Cell 2018;33:843-52.e4.

20. Keenan TE, Burke KP, Van Allen EM. Genomic correlates of response to immune checkpoint blockade. Nat Med 2019;25:389-402.

21. Mezquita L, Auclin E, Ferrara R, et al. Association of the Lung Immune Prognostic Index With Immune Checkpoint Inhibitor Outcomes in Patients With Advanced Non-Small Cell Lung Cancer. JAMA Oncol 2018;4:351-7.

22. Martini DJ, Liu Y, Shabto JM, et al. Novel Risk Scoring System for Patients with Metastatic Renal Cell Carcinoma Treated with Immune Checkpoint Inhibitors. Oncologist 2020;25:e484-91.

23. Eisenhauer EA, Therasse P, Bogaerts J, et al. New response evaluation criteria in solid tumours: revised RECIST guideline (version 1.1). Eur J Cancer 2009;45:228-47.

24. Rizvi NA, Hellmann MD, Snyder A, et al. Cancer immunology. Mutational landscape determines sensitivity to PD-1 blockade in non-small cell lung cancer. Science 2015;348:124-8.

25. Rizvi H, Sanchez-Vega F, La K, et al. Molecular Determinants of Response to Anti-Programmed Cell Death (PD)-1 and Anti-Programmed Death-Ligand 1 (PD-L1) Blockade in Patients With Non-Small-Cell Lung Cancer Profiled With Targeted Next-Generation Sequencing. J Clin Oncol 2018;36:633-41.

26. Havel JJ, Chowell D, Chan TA. The evolving landscape of biomarkers for checkpoint inhibitor immunotherapy. Nat Rev Cancer 2019;19:133-50.

27. Quail DF, Joyce JA. Microenvironmental regulation of tumor progression and metastasis. Nat Med 2013;19:1423-37.

28. Tamiya M, Tamiya A, Inoue $T$, et al. Metastatic site as a predictor of nivolumab efficacy in patients with advanced non-small cell lung cancer: A retrospective multicenter trial. PLoS One 2018;13:e0192227.

29. Kang DH, Chung C, Kim JO, et al. Pleural or pericardial metastasis: A significant factor affecting efficacy and adverse events in lung cancer patients treated with PD-1/ PD-L1 inhibitors. Thorac Cancer 2018;9:1500-8.

30. Mikubo M, Nakashima H, Naito M, et al. Prognostic impact of uncertain parietal pleural invasion at adhesion sites in non-small cell lung cancer patients. Lung Cancer 2017;108:103-8.

31. Lee DS, Kang JH, Lee CG, et al. Predicting Survival in Patients with Advanced Non-squamous Non-small Cell
Lung Cancer: Validating the Extent of Metastasis. Cancer Res Treat 2013;45:95-102.

32. Kuchuk M, Kuchuk I, Sabri E, et al. The incidence and clinical impact of bone metastases in non-small cell lung cancer. Lung Cancer 2015;89:197-202.

33. Kuijpers C, Hendriks LEL, Derks JL, et al. Association of molecular status and metastatic organs at diagnosis in patients with stage IV non-squamous non-small cell lung cancer. Lung Cancer 2018;121:76-81.

34. Na, II, Park JH, Choe du H, et al. Association of epidermal growth factor receptor mutations with metastatic presentations in non-small cell lung cancer. ISRN Oncol 2011;2011:756265.

35. Sholl LM, Aisner DL, Varella-Garcia M, et al. Multiinstitutional Oncogenic Driver Mutation Analysis in Lung Adenocarcinoma: The Lung Cancer Mutation Consortium Experience. J Thorac Oncol 2015;10:768-77.

36. Garassino MC, Cho BC, Kim JH, et al. Durvalumab as third-line or later treatment for advanced non-small-cell lung cancer (ATLANTIC): an open-label, single-arm, phase 2 study. Lancet Oncol 2018;19:521-36.

37. Sharma P, Wagner K, Wolchok JD, et al. Novel cancer immunotherapy agents with survival benefit: recent successes and next steps. Nat Rev Cancer 2011;11:805-12.

38. Pine JK, Morris E, Hutchins GG, et al. Systemic neutrophil-to-lymphocyte ratio in colorectal cancer: the relationship to patient survival, tumour biology and local lymphocytic response to tumour. Br J Cancer 2015;113:204-11.

39. Balkwill F, Mantovani A. Inflammation and cancer: back to Virchow? Lancet 2001;357:539-45.

40. McMillan DC. The systemic inflammation-based Glasgow Prognostic Score: a decade of experience in patients with cancer. Cancer Treat Rev 2013;39:534-40.

41. Petrelli F, Cabiddu M, Coinu A, et al. Prognostic role of lactate dehydrogenase in solid tumors: a systematic review and meta-analysis of 76 studies. Acta Oncol 2015;54:961-70.

42. Hellmann MD, Ciuleanu TE, Pluzanski A, et al. Nivolumab plus Ipilimumab in Lung Cancer with a High Tumor Mutational Burden. N Engl J Med 2018;378:2093-104.

43. Hu-Lieskovan S, Lisberg A, Zaretsky JM, et al. Tumor Characteristics Associated with Benefit from Pembrolizumab in Advanced Non-Small Cell Lung Cancer. Clin Cancer Res 2019;25:5061-8.

44. Kazandjian D, Gong Y, Keegan P, et al. Prognostic Value of the Lung Immune Prognostic Index for Patients Treated for Metastatic Non-Small Cell Lung Cancer. JAMA Oncol. 2019;5:1481-5. 
45. Sorich MJ, Rowland A, Karapetis CS, et al. Evaluation of the Lung Immune Prognostic Index for Prediction of Survival and Response in Patients Treated With Atezolizumab for NSCLC: Pooled Analysis of Clinical Trials. J Thorac Oncol 2019;14:1440-6.

46. Mazzaschi G, Minari R, Zecca A, et al. Soluble PDL1 and Circulating CD8+PD-1+ and NK Cells Enclose a Prognostic and Predictive Immune Effector Score in Immunotherapy Treated NSCLC patients. Lung Cancer 2020;148:1-11.

Cite this article as: Li C, Shi M, Lin X, Zhang Y, Yu S, Zhou C, Yang N, Zhang J, Zhang F, Lv T, Liu H, Song Y. Novel risk scoring system for immune checkpoint inhibitors treatment in non-small cell lung cancer. Transl Lung Cancer Res 2021;10(2):776-789. doi: 10.21037/tlcr-20-832
47. Kasahara N, Sunaga N, Tsukagoshi Y, et al. Post-treatment Glasgow Prognostic Score Predicts Efficacy in Advanced Non-small-cell Lung Cancer Treated With Anti-PD1. Anticancer Res 2019;39:1455-61.

48. Dolan RD, Daly L, Sim WMJ, et al. Comparison of the prognostic value of ECOG-PS, mGPS and BMI/WL: Implications for a clinically important framework in the assessment and treatment of advanced cancer. Clin Nutr 2020;39:2889-95. 